\title{
Desafios para a pesquisa sobre as dimensões humanas das mudanças ambientais globais: um olhar latino-americano*
}

\author{
Challenges to the Research about the Human \\ Dimensions of Global Environmental Changes: \\ a Latin American point-of-view
}

\author{
Rafael D'Almeida MARTINS** \\ Leila da Costa FERREIRA***
}

\begin{abstract}
RESUMO
Os estudos ambientais desenvolveram-se lentamente no interior das ciências sociais latino-americanas. Contudo, as duas últimas décadas foram marcadas por uma expressiva expansão da pesquisa e do ensino da temática ambiental na região. Nesse sentido, os prováveis impactos das mudanças ambientais globais penetraram rapidamente os debates contemporâneos sobre ambiente e sociedade, primeiramente nos países industrializados e, posteriormente, no mundo em desenvolvimento, incluindo a América Latina. Este artigo busca contribuir para essa discussão fornecendo uma reflexão sobre o estado da arte e os desafios para o desenvolvimento e para a consolidação da pesquisa sobre as dimensões humanas das mudanças ambientais globais na América Latina. Por meio de uma investigação sobre a produção científica da região, que incluiu a sistematização e a compilação de um banco de dados, buscou-se entender a produção intelectual e o desenvolvimento deste campo em países latino-americanos a partir de análises de autores como D. J. Hogan e L. C. Ferreira. Argumenta-se que, embora seja possível identificar uma literatura emergente na região, o envolvimento das ciências sociais latino-americanas ainda é tímido e tentativo.

Palavras-chave: dimensões humanas; mudanças ambientais globais; América Latina; ambiente e sociedade; mudança climática.
\end{abstract}

\begin{abstract}
The environmental field has developed slowly within social sciences in Latin America. However, the last two decades have been marked by the expansion of research and teaching activities in the environmental area. In this sense, the likely impact of global environmental changes have rapidly penetrated
\end{abstract}

\footnotetext{
"Este texto é dedicado ao Prof. Daniel Joseph Hogan (in memoriam).

"* Administrador público pela Escola de Administração de Empresas de São Paulo da Fundação Getúlio Vargas (FGV/EAESP). Doutorando em Ambiente e Sociedade no Núcleo de Estudos e Pesquisas Ambientais (NEPAM) da Universidade Estadual de Campinas (UNICAMP). Email: rdamartins@gmail.com

*** Doutora em Ciências Sociais pela Universidade Estadual de Campinas (UNICAMP). Professora Titular do Instituto de Filosofia e Ciências Humanas (IFCH) e do Núcleo de Estudos e Pesquisas Ambientais (NEPAM) da UNICAMP. Coordenadora do Programa de Doutorado em Ambiente e Sociedade. Email: leilacf@unicamp.br
} 
the contemporary debates on environment and society, primarily in developed countries and later on in developing countries, including Latin America. This paper seeks to contribute to the discussion by providing an overview and identifying the challenges for the development and consolidation of the research on the human dimensions of global environmental change. Based on the assessment and systematization of the intellectual production, this paper seeks to understand this field in Latin America, building upon the contribution of authors such as D.J. Hogan and L.C. Ferreira. The paper argues that although it is possible to identify an emergent body of literature in the region, the involvement of Latin American social science in the human dimension research is still timid and tentative.

Key-words: human dimensions; global environmental change; Latin America; environment and society; climate change.

\section{Introdução}

A pesquisa científica sobre a interface entre ambiente e sociedade está em rápida evolução em todo o mundo e desenvolve-se por meio da contribuição de diferentes áreas de especialização disciplinar e do conhecimento ${ }^{1}$. Nas ciências sociais ${ }^{2}$, os estudos ambientais desenvolveram-se lentamente tanto no Brasil quanto na América Latina (DRUMMOND, 2006; FERREIRA, 2006a; HOGAN, 2007, 2009). Todavia, a emergência dos movimentos ambientalistas da década de 1960 e os questionamentos sobre os impactos sociais da destruição e da poluição do meio ambiente, somados às possibilidades de desenvolvimento com sustentabilidade, colocaram a temática do meio ambiente na agenda das ciências sociais da região (FERREIRA, 2006a; HOGAN, 2007).

No início, o objetivo era identificar os componentes ambientais de uma sociedade injusta e desigual (HOGAN, 2007). Ao contrário da experiência de muitos países industrializados, a preocupação dos cientistas sociais com os ecossistemas e a preservação ambiental surgiu tardiamente e a atenção dada à biodiversidade e às áreas com grande importância ecológica emergiu com os impactos da degradação ambiental na manutenção dos modos de vida de populações tradicionais (HOGAN; TOMALSQUIM, 2001).

Particularmente no interior das ciências sociais, a temática ambiental tem sido objeto de várias análises e revisões conceituais e metodológicas (DUNLAP; CATTON, 1979; LOWE; RUDIG, 1986; BUTTEL, 1987, 1996, 1997; HANNIGAN, 1995; PULIDO, 1996; DUNLAP, 1997; REDCLIFT; WOODGATE, 1997; FERREIRA, 1997, 2006a; MOL; SPAARGAREN, 2000; LIMA; PORTILHO, 2001; DRUMMOND, 2006). Nesse sentido, é possível notar que a pesquisa científica sobre a interação entre ambiente e sociedade inclui uma grande variedade de temas, sendo possível notar um incremento de suas atividades ao longo dos anos. Os resultados desses esforços buscam dar respostas a crescentes demandas por estudos interdisciplinares no interior dessa literatura e produção intelectual (FERREIRA, 2006a; FERREIRA et al., 2006a, 2006b; FERREIRA et al., 2007a, 2007b; MARTINS; FERREIRA, 2009, 2010; FERREIRA, no prelo). No entanto, alguns autores argumentam que a inclusão das questões ambientais nas ciências sociais ainda não resultou em um novo paradigma. Assim, ao invés de um campo consolidado de estudos ambientais, essas questões criaram subáreas de investigação, distribuídas de acordo com diferentes abordagens teóricas e metodológicas (ALONSO; COSTA, 2002; FERREIRA, 2006a).

Ao mesmo tempo, o conceito de risco vinculado às preocupações ambientais passou a ocupar uma posição de destaque na teoria social com os trabalhos seminais de Beck (1992, 1999) e Giddens (1991), que consideraram os riscos ambientais e tecnológicos como categorias analíticas fundamentais para o entendimento da modernidade (FERREIRA, 2006a). Neste contexto, a temática das Mudanças Ambientais Globais (MAG) penetrou os debates acadêmicos e políticos contemporâneos, baseando-se em crescentes evidências científicas que reafirmavam o risco dessas mudanças para a sociedade em geral, sobretudo aquelas que vêm alterando o Sistema Climático Global por meio do aquecimento da superfície da Terra.

Apesar de grandes incertezas e de posições acadêmicas divergentes, as MAG sensibilizam há várias décadas

\footnotetext{
${ }^{1}$ Entre as áreas mais expressivas estão biologia, ecologia, economia, demografia, geografia, ciência política e sociologia.

${ }^{2}$ Neste artigo, o termo ciências sociais abrange um vasto leque de atividades de pesquisa geralmente associadas a disciplinas como economia, sociologia, ciência política, antropologia, geografia e história, além de campos interdisciplinares, tais como ciências ambientais, demografia, ecologia humana e administração.
} 
parcela importante da comunidade científica, sobretudo dos países industrializados ${ }^{3}$. De forma mais recente, também se observa uma crescente movimentação de pesquisadores dedicados a essas questões nos países considerados de economia emergente. Nesse sentido, a atenção dada às medidas destinadas a mitigar a emissão dos Gases de Efeito Estufa (GEEs) - gases considerados por muitos como um importante vetor do aquecimento global - e o desafio de adaptar-se aos impactos das alterações climáticas em andamento foi, assim, aumentando gradualmente e introduzindo novos referenciais para o debate e para a reflexão dos temas posicionados na interface entre meio ambiente e sociedade (MARTINS; FERREIRA, 2009, 2010; HOGAN, 2009).

No centro do debate, estão atividades humanas que são responsáveis por muitas mudanças ambientais de larga escala. Tais mudanças vão desde a do clima do planeta, como foi mencionado acima, até os impactos cumulativos da perda de diversidade biológica, mudanças no uso e na ocupação do solo, poluição química, escassez de recursos hídricos, entre outras (MEA, 2005; IHDP, 2007; IPCC, 2007; ROCKSTRÖM et al., 2009). Dessa forma, abordar essas grandes questões e encontrar formas de promover o desenvolvimento sustentável exige um esforço concertado e compartilhado de pesquisadores que se concentram em um amplo conjunto de linhas de pesquisa que consideram em seus estudos não somente a análise de componentes e aspectos biogeoquímicos da Terra, mas também as várias dimensões do comportamento humano.

Nessa linha, o Intergovernmental Panel on Climate Change (IPCC), o National Research Council (NRC), nos Estados Unidos da América (EUA), e o Millennium Ecosystem Assessment (MEA), entre outros organismos científicos internacionais, têm sistematicamente avaliado e organizado a produção científica sobre mudanças ambientais globais, ressaltando a necessidade de um engajamento mais robusto por parte das ciências sociais nesse debate. Busca-se com esse engajamento aumentar a compreensão sobre os aspectos sociais desses problemas marcados fortemente pelas dimensões políticas, econômicas e ambientais. Como consequência, um conjunto de projetos ${ }^{4}$ foi levado adiante por pesquisadores e tomadores de decisão como forma de compreender melhor a contribuição das ciências sociais no entendimento dessas questões, mapeando algumas das iniciativas ad hoc existentes. Espera-se que a constante reflexão sobre essa contribuição e produção científica continue estimulando o envolvimento necessário das ciências sociais nessa discussão.

Todavia, no contexto da América Latina, esses esforços ainda são escassos e incipientes, sendo necessário um maior número de trabalhos para apontar lacunas e apoiar a formulação de estratégias que possibilitem abordagens que sejam capazes de enfrentar os desafios colocados pelas MAG no século XXI. Dada a importância da biodiversidade regional, somada aos atributos ecológicos de vários dos países latino-americanos, torna-se necessário discutir o estado da arte da investigação sobre as dimensões humanas das MAG na região, bem como apontar os desafios para o seu desenvolvimento considerando o contexto em que a América Latina está inserida.

A partir de uma leitura da experiência acumulada no plano internacional, este artigo objetiva apresentar uma discussão sobre essas pesquisas na América Latina, lançando luz não somente no que pode ser considerado seu estado da arte, mas também sobre os desafios para um maior envolvimento das ciências sociais nesses esforços. Nesse sentido, a reflexão desenvolvida neste trabalho baseou-se em duas questões centrais. A primeira remete aos principais temas que são objetos de estudo por parte da comunidade científica da região, bem como nos aspectos condicionantes dessa produção acadêmica e intelectual em termos da própria especificidade regional. Os resultados deste levantamento permitem levar adiante uma discussão sobre o nível de institucionalização dessas temáticas nos interior dos países latino-americanos investigados. A segunda questão teve como indagação os desafios para o desenvolvimento e a evolução da pesquisa sobre as Dimensões Humanas das Mudanças Ambientais Globais (DHMAG).

$\mathrm{O}$ debate sobre esses desafios, que incluem tanto barreiras como potenciais identificados, contribui com uma área do qual grande parte dos países latino-americanos apresenta pouco acúmulo e tradição (HOGAN, 2007; MARTINS; FERREIRA, 2009, 2010). Nesse sentido, este artigo

\footnotetext{
${ }^{3}$ Pode-se citar como exemplo de um desses primeiros esforços uma declaração feita por um grupo formado por mais 300 cientistas renomados durante uma conferência mundial realizada em 1988, na cidade de Toronto, Canadá. Segundo este grupo, "Humanity is conducting an unintended, uncontrolled, globally pervasive experiment whose ultimate consequences could be second only to a global nuclear war. The Earth's atmosphere is being changed at an unprecedented rate by pollutants resulting from human activities, inefficient and wasteful fossil fuel use and the effects of rapid population growth in many regions" (The Changing Atmosphere: Implications for Global Security, Conference Statement. Documentation et Rapports de la Conference).

${ }^{4}$ Um exemplo relevante é o Programa Exploring the Social Dimension of Climate Change do Banco Mundial.
} 
une-se a uma série de esforços para avaliar o estado atual desses temas, uma vez que parte integrante desta agenda de investigação é o próprio estudo da pesquisa sobre as MAG, reconhecendo que o processo de fazer ciência é uma atividade inerentemente social (GALLOPÍN et al., 2001; JANSSEN et al., 2006; BIERMANN et al., 2009). Dessa forma, o artigo busca compreender de forma específica o desenvolvimento dessa agenda de pesquisa nos países da América Latina como estratégia para identificar o que é essencial para apoiar um envolvimento mais robusto e sistemático das ciências sociais na investigação sobre as DHMAG.

O artigo está organizado da seguinte forma: em primeiro lugar, apresenta-se uma nota metodológica, seguida por uma tentativa de definição das DHMAG baseando-se na experiência internacional. Em seguida, discorre-se sobre o estado da arte dessas pesquisas na América Latina e no Brasil a partir da análise de nossa compilação. $\mathrm{Na}$ última seção, discutem-se alguns desafios identificados para a pesquisa sobre DHMAG na América Latina tendo como base as lacunas encontradas a partir aplicação da abordagem metodológica, seguida pelas considerações finais do trabalho. Argumenta-se que, embora seja possível identificar uma emergente literatura sobre a temática das DHMAG na região, o envolvimento das ciências sociais latino-americanas nesse debate ainda é tímido e, muitas vezes, tentativo. A evidência mostra que os papéis desempenhados pela cooperação entre países e alguns programas internacionais de pesquisa, bem como um papel ativo por parte das agências de financiamento, têm sido fundamentais para garantir as atividades de pesquisa sobre as DHMAG, induzindo um engajamento crescente das ciências sociais latino-americanas na discussão em questão.

\section{Nota metodológica}

Este artigo é resultado de um esforço maior de sistematizar e avaliar a produção intelectual sobre a questão ambiental no interior das ciências sociais latino-americanas, buscando analisar os aportes conceituais e metodológicos dessa produção acadêmica sobre temas na interface entre ambiente e sociedade, investigando como especificidades dessa literatura decorrem (ou não) de particularidades socioeconômicas e socioambientais desses países ${ }^{5}$.

A fim de sistematizar a pesquisa sobre as DHMAG na América Latina, organizou-se uma compilação manual por meio da revisão sistemática de artigos e publicações sobre temas relacionados à temática em questão. Esses dados foram organizados na forma do Banco de Dados Latino-americano em Ambiente e Sociedade (LAMAS). O objetivo principal deste banco de dados foi investigar os principais temas de pesquisa que estão inseridos na pequena comunidade de pesquisadores dedicados à análise das DHMAG.

Nesse sentido, criou-se um conjunto de palavras-chave cobrindo as principais áreas de investigação (Tabela 1). Título e resumo de todos os documentos foram selecionados por palavras-chave e verificados manualmente. Somente as publicações consideradas na área das DHMAG foram mantidas, abrangendo o período de 2001 a $2008^{6}$. A decisão de incluir ou excluir uma publicação no banco de dados foi baseada nas informações fornecidas no título e no resumo da publicação, seguindo a abordagem metodológica proposta por Janssen et al. (2006).

Ressalta-se que não é objetivo desse presente artigo fornecer uma coletânea exaustiva da produção acadêmica sobre DHMAG, incluindo todos seus autores e publicações, mas sim fornecer os subsídios para uma visão ampla do seu processo de desenvolvimento e institucionalização. Nesse sentido, não foram feitas menções a autores ou a trabalhos específicos, uma vez que essas fogem do escopo e da proposta desta análise.

A maioria da produção científica nessa área está publicada em revistas científicas latino-americanas e internacionais arbitradas, além da presença minoritária de publicações na forma de livro. Para efeito dessa compilação, foram considerados apenas os documentos que tinham como primeiro autor um pesquisador vinculado a instituições latino-americanas.

Também vale lembrar que este trabalho concentra-se nas ciências humanas com foco em disciplinas que estudam a interação entre ambiente e sociedade, mais do que nos aspectos naturais e biogeofísicos de processos de mudança

\footnotetext{
${ }^{5}$ Trata-se do projeto “A questão ambiental, Interdisciplinaridade, teoria social e produção intelectual na América Latina” (Proc. 2005/52317-1), apoiado pela Fundação de Amparo à Pesquisa do Estado de São Paulo (FAPESP) e coordenado pela Profa. Leila da Costa Ferreira (NEPAM/IFCH/UNICAMP).

${ }^{6}$ É importante notar que nos anos de 2009 e 2010, após a divulgação do Quarto Relatório Síntese do IPCC, foram publicados vários trabalhos que poderiam fazer parte desta compilação. Entretanto, dado o recorte temporal determinado previamente, essas publicações não integram a análise em questão.
} 
TABELA 1 - PALAVRAS-CHAVE UTILIZADAS PARA COMPILAÇÃO E LEVANTAMENTO DA PRODUÇÃO ACADÊMICA SOBRE DHMAG (EM INGLÊS)

\begin{tabular}{|c|c|}
\hline \multirow{26}{*}{ 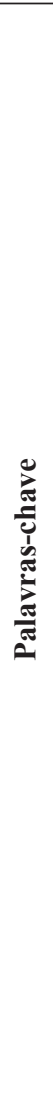 } & Adaptation \\
\hline & Adaptive capacity \\
\hline & Adaptive response \\
\hline & Climate change \\
\hline & Climate change \\
\hline & Climate change impacts \\
\hline & Deforestation \\
\hline & Desertification \\
\hline & Environmental change \\
\hline & Food security \\
\hline & Global change \\
\hline & Global climate change \\
\hline & Global environmental change \\
\hline & Global warming \\
\hline & Human adaptation \\
\hline & $\begin{array}{l}\text { Human dimensions of global environmental } \\
\text { change }\end{array}$ \\
\hline & Human security \\
\hline & Land cover \\
\hline & Land use change \\
\hline & Mitigation \\
\hline & Resilience \\
\hline & Social adaptability \\
\hline & Social adaptation \\
\hline & Vulnerability \\
\hline & Urbanization \\
\hline & Water resources management \\
\hline
\end{tabular}

FONTE: MARTINS; FERREIRA (2009; 2010).

ambiental. Assim, não foi incluída nesse levantamento a abundante literatura desenvolvida pelas ciências naturais em geral, que claramente apresentam uma maior tradição no estudo e na análise dos temas correlatos às MAG.

Outro aspecto importante que merece destaque baseia-se no fato que a seleção dos trabalhos levou em consideração a necessidade de capturar o componente "global" dos processos de mudança ambiental investigado pelos trabalhos científicos. Assim, a tarefa de extrair esse elemento de publicações foi difícil, já que muitas vezes essas tratavam apenas de problemas ou questões claramente locais (ou nacional), com pouca ou nenhuma interação com as escalas superiores de análise (regional e global). O método empregado possibilitou uma avaliação em profundidade que contribuiu para o entendimento de vários aspectos relacionados aos desafios da pesquisa sobre as DHMAG na região.

\section{Mudanças ambientais globais: uma aproximação}

Definições de MAG variam consideravelmente e podem ser interpretadas em diferentes contextos. No entanto, é consenso que elas correspondem à alterações no ambiente físico e biogeoquímico - incluindo a Terra, os oceanos e a atmosfera - causadas por fenômenos naturais ou induzidas por atividades humanas como o desmatamento, o consumo de combustíveis fósseis, a urbanização, o uso do solo, a atividade agrícola intensiva, a extração de água doce, a sobre-exploração da pesca e a produção de resíduos sólidos (IGBP, 2001). Podem se manifestar por meio de alterações climáticas, da destruição da camada de ozônio, da perda de biodiversidade, de mudanças em sistemas hidrológicos e da degradação do solo, cujos efeitos são sentidos em muitos processos que são fundamentais para a vida humana como, por exemplo, os relacionados aos sistemas de produção de alimentos (BIERMANN et al., 2009). Essas mudanças também se referem ao conjunto de interações entre processos físicos, químicos, biológicos e humanos que transportam e transformam materiais e energia que são fundamentais para a manutenção da vida no planeta (IGBP, 2001; STEFFEN et al., 2004; ROCKSTRÖM et al., 2009).

\section{As dimensões humanas dos processos de mudança ambiental}

Há um intenso debate na literatura sobre o que constitui a pesquisa sobre as dimensões humanas dessas mudanças (MARTINS; FERREIRA, 2009, 2010). Por exemplo, o International Human Dimensions Programme on Global Environmental Changes (IHDP) define as DHMAG como sendo " $[\ldots]$ the set of biophysical transformations of land, oceans and atmosphere, driven by an interwoven system of human activities and natural processes" (IHDP, 2007, p. 19). Esta definição abrange as causas e as consequências não só de ações individuais, mas também coletivas, que incluem as alterações que levam à transformação de sistemas físicos e biológicos do planeta (IGBP, 2001; STEFFEN et al., 2004; BIERMANN et al., 2009; ROCKSTRÖM et al., 2009). 
Essas alterações têm a capacidade de afetar a qualidade de vida dos seres humanos, bem como de reduzir as oportunidades de desenvolvimento sustentável na escala global.

Assim colocado, a pesquisa sobre DHMAG neste artigo corresponde ao estudo das "atividades humanas que alteram o ambiente da Terra e das motivações dessas atividades, suas consequências para diferentes sociedades e economias e como pessoas e instituições respondem aos impactos e a expectativa dessas mudanças" (NRC, 1999a, p. 293). Nesse sentido, essa pesquisa envolve a análise de diversos processos sociais, políticos e econômicos, bem como as instituições que influenciam o comportamento e as decisões humanas relativas a esses processos (NRC, 1999b).

Em suma, a pesquisa sobre as DHMAG visa compreender como as atividades humanas impactam as emissões de GEE, a qualidade do ar, a mudança de cobertura e uso do solo e as alterações em ecossistemas terrestres e marinhos, entre outras. Por meio dela, é possível prever o curso das atividades que levam a essas transformações, estimar como mudanças no clima, na cobertura e uso do solo, nos ecossistemas e na atmosfera poderão afetar a produção de alimentos, a disponibilidade de recursos hídricos, a saúde humana e a economia de países e setores, além de possibilitar a análise de diferentes formas de governança de recursos naturais e da viabilidade e custos de transformações tecnológicas, econômicas e políticas para enfrentar essas mudanças. Nesse sentido, a pesquisa sobre as DHMAG constrói conhecimentos básicos da interação entre população e ambiente, fornecendo informações e ferramentas importantes para tomadores de decisão e a sociedade em geral (HOGAN; MARANDOLA JR., 2009).

Apesar da longa trajetória de pesquisas sobre os aspectos sociais e políticos da mudança ambiental, a investigação sobre as dimensões humanas dessas mudanças só foi formalmente associada à comunidade científica dedicada às MAG no final da década de 1980 (NRC, 1999a). Atualmente, é consenso entre essa comunidade que as MAG não poderão ser entendidas, muito menos enfrentadas de forma adequada, sem as contribuições das ciências sociais e da análise dos aspectos socioeconômicos, políticos e culturais desses processos (HOGAN, 2007; IHDP, 2007; BIERMANN et al., 2009).

Ao longo das últimas duas décadas, pesquisadores dedicados às dimensões humanas vêm tentando entender as atividades humanas que levam à mudança ambiental no nível global e como elas variam ao longo do espaço, tempo e demais escalas, bem como quais as consequências e os impactos dessas mudanças para a agricultura, a saúde, as zonas costeiras e os diversos setores econômicos, por exemplo. Destaca-se que nos últimos anos houve significativo progresso em muitas questões importantes, como as causas sociais do desmatamento em regiões da Amazônia, o papel das dimensões sociais, políticas, econômicas e culturais nas decisões sobre o uso e a ocupação do solo, bem como suas relações com as populações locais (LAHSEN; NOBRE, 2007). Também tiveram avanços expressivos na capacidade de combinar dados sociais e geofísicos no âmbito de Sistemas de Informação Geográfica (SIG), que são crescentemente aplicados para a compreensão de como processos eminentemente locais estão interligados nas escalas nacional, regional e global (BRONDIZIO; MORAN, 2008).

Nesse contexto, as primeiras demandas para a participação das ciências sociais nessa área de pesquisa vieram da comunidade científica ligada às ciências naturais que viram antecipadamente que as atividades humanas eram um dos principais vetores da mudança ambiental e climática (HOGAN, 2007; HOGAN; MARANDOLA JR., 2009) e as primeiras iniciativas de pesquisadores das ciências sociais nessas iniciativas incluíram múltiplas abordagens metodológicas para o início do processo de formulação de uma agenda de pesquisa (URICH et al., 2005).

\section{A experiência internacional}

Pode-se afirmar que existe um nível importante de atividade científica no campo das MAG incluindo o relativo sucesso do estudo de suas dimensões humanas (URICH et al., 2005; HOGAN, 2007). Exemplos dessas atividades científicas são o International Geosphere-Biosphere Programme (IGBP), o World Climate Research Programme (WCRP), o IPCC e o MEA, que têm apresentado um alto grau de cooperação internacional entre pesquisadores das ciências biológicas, atmosféricas e das ciências do sistema terrestre em geral. Desde o início dos debates contemporâneos sobre o aquecimento global e a destruição da camada de ozônio, existe crescente interesse nas implicações sociais dessas alterações, uma vez que a população é muitas vezes vista tanto como a causa dessas mudanças, como também como principais vítimas das consequências desses fenômenos (WILBANKS; KATES, 1999).

Dessa maneira, no início da década de 1990, houve uma tentativa do International Social Sciences Council (ISSC) para desenvolver um Programa sobre as Human 
Dimensions (HDP), paralelamente à experiência do IGBP e do WCRP, porém com sucesso apenas limitado. Mais recentemente, em 1996, esse interesse foi renovado e uma nova versão do HDP foi criada para desenvolver o trabalho que havia sido originalmente pensado para o programa anterior, criando-se assim o International Human Dimensions Programme (IHDP). Na mesma direção, o IPCC reconheceu a necessidade de considerar mais fortemente as dimensões humanas nos seus relatórios de avaliação a partir dos Terceiro e Quarto Relatórios Síntese (TAR e AR4, respectivamente), assim como o United Nations Environment Programme (UNEP) para a preparação do seu relatório Global Environment Outlook (URICH et al., 2005).

Segundo Hogan (2007), houve duas iniciativas relacionadas que foram importantes para o estabelecimento de uma agenda científica, além do intercâmbio e da divulgação dos resultados de pesquisa sobre as DHMAG. Essas iniciativas citadas pelo autor foram o Open Meeting of the Human Dimensions of Global Environmental Change Research Community ${ }^{7}$ e a participação ativa das Academias Nacionais de Ciências (National Academy of Sciences). Em muitos países, estas Academias estabeleceram comitês nacionais e mobilizaram linhas e programas de financiamento específicos para apoiar pesquisas e eventos na direção de uma maior compreensão sobre as DHMAG. Juntas, essas duas iniciativas foram responsáveis por parcela significativa dos trabalhos sobre as DHMAG que estão disponíveis até o momento no Brasil e na América Latina (HOGAN, 2007).

\section{A pesquisa sobre as dimensões humanas na Amé- rica Latina}

Embora as ciências sociais tenham evoluído de forma diferente nos países latino-americanos, atribui-se à década de 1960 o momento em que os cientistas sociais começaram a dar maior atenção aos problemas ambientais (FERREIRA, 1997, 2006a). Nesta região, a institucionalização do campo de pesquisa sobre ambiente e sociedade, de caráter claramente interdisciplinar, começou posteriormente, entre os anos de 1980 e 1990, e avaliações preliminares sobre a produção intelectual da América Latina demonstram a adoção de diferentes abordagens teóricas e metodológicas para a compreensão desses problemas nos diferentes contextos (FERREIRA, 2006b; FERREIRA et al., 2006a, 2006b; FERREIRA et al., 2007a, 2007b; FERREIRA, no prelo).

Hogan (2007) afirma que, apesar de muitos cientistas sociais terem começado a estudar os aspectos sociais e as consequências da mudança ambiental, muitos estavam "despreparados para incorporar os desafios globais no âmbito do seu trabalho" (HOGAN, 2007, p. 161). Apesar de vários fatores serem responsáveis para esse despreparo, um ponto importante que merece ser destacado baseia-se no fato que os efeitos das MAG, sejam eles a elevação do nível médio dos mares, entre outros, ocorrem em escalas temporais e espaciais que não são refletidas nos paradigmas de pesquisa das ciências sociais em geral. As escalas de análise nas ciências sociais são diferentes daquelas costumeiramente adotadas pelas ciências naturais, onde as bases conceituais e empíricas são, de maneira geral, aquelas onde as MAG podem ser melhores observadas (HOGAN; TOMALSQUIM, 2001).

Apesar de não tratarem especificamente da questão ambiental, Bertero et al. (1998) defendem que uma temática necessita de paradigmas para consolidá-la como um campo de investigação, utilizando como base as explicações de Kuhn sobre a "ciência normal". Para esses autores, é o paradigma que permite a construção e o desenvolvimento de um "protocolo científico" na área de pesquisa em destaque.

Na América Latina, em contraste com a experiência de outros campos científicos, as ciências sociais não iniciaram a investigação sobre o meio ambiente através do desenvolvimento gradual de seus paradigmas de pesquisa, uma vez que foi o surgimento do movimento ambientalista, combinado com os graves problemas ambientais da região, que colocaram a questão ambiental na agenda de pesquisa das ciências sociais latino-americanas (HOGAN, 2007).

Ao analisar a literatura da ciência social ambiental da região, que busca analisar os problemas ambientais latino-americanos, é evidente que a maior preocupação dos estudos concentra-se nos níveis local e nacional e temas relacionados às MAG estão longe de ser considerados uma prioridade para a maioria dos cientistas sociais. Neste contexto, pode-se argumentar que as DHMAG como uma área de investigação apresentam um atraso quando comparadas ao acúmulo disponível no interior das ciências naturais e exatas (DRUMMOND, 2006; HOGAN, 2007, 2009), apesar

${ }^{7}$ O primeiro foi organizado na Duke University (EUA), em 1995. Posteriormente, ele foi seguido de reuniões na Áustria (1997), Japão (1999), Brasil (2001), Canadá (2003), Alemanha (2005) e, novamente, Alemanha (2009). 
de interessantes exemplos recentes disponíveis na literatura internacional que buscam articular as duas perspectivas de pesquisa (i.e. GRANT et al., 2002).

Nesse sentido, a análise da compilação organizada para servir de base para este artigo mostra que é possível identificar um incremento nos últimos anos do número de estudos e publicações que podem ser considerados como pertencendo à área das DHMAG, uma vez que essa questão vem recebendo mais atenção da mídia e penetrando rapidamente os debates científicos e políticos contemporâneos, sobretudo após a divulgação do Fourth Assessment Report (AR4) do IPCC, em abril de 2007. O lançamento desse conjunto de relatórios síntese contou com grande notoriedade não só por parte dos veículos de divulgação especializados, como também os de grande alcance para o público em geral, contribuindo para aumentar o interesse da sociedade (GIDDENS, 2009; HOGAN, 2009; MARTINS; FERREIRA, 2009, 2010).

Outro ponto que merece destaque recai sobre a criação de um número importante de programas interdisciplinares de pós-graduação enfocando a interface entre ambiente e sociedade, fato esse que pode ser considerado relativamente novo na região, mesmo considerando que alguns países estão mais avançados do que outros na pesquisa sobre as DHMAG, já que contam com linhas e programas de pesquisa mais consolidados estimulando um diálogo profícuo entre as ciências sociais com as variadas abordagens sobre as MAG (HOGAN, 2007, 2009; FERREIRA, no prelo).

\section{Um panorama atual da pesquisa sobre as DHMAG}

Em análises anteriores sobre a pesquisa sobre as DHMAG na América Latina, autores como Martins e Ferreira $(2009,2010)$ mostraram que países como Argentina, Bolívia, Brasil, Chile, México e Venezuela ${ }^{8}$ apresentam produção acadêmica no campo em questão. A análise do conjunto de dados compilados mostrou que os temas explorados por esses pesquisadores latino-americanos variam consideravelmente e levam em consideração não só especificidades de cada país, mas também da região (FERREIRA, 2006a, 2006b; FERREIRA et al., 2006a, 2006b; FERREIRA et al., 2007a, 2007b).
Esses trabalhos são resultados da contribuição de diferentes disciplinas das ciências sociais, incluindo geografia, demografia, antropologia, sociologia, ciência política, relações internacionais e economia. Também nota-se que parcela considerável da produção acadêmica foi realizada em parceria com pesquisadores e centros de pesquisa de países desenvolvidos, especialmente dos EUA e Europa. Nesse sentido, o levantamento dos trabalhos analisados não identificou colaboração com outras regiões do mundo como África e Ásia (MARTINS; FERREIRA, 2009, 2010).

Avaliações anteriores sobre a pesquisa das DHMAG têm mostrado que é mais fácil envolver cientistas sociais tendo como ponto de partida temas que já são objeto de investigação desses pesquisadores em contraponto à introdução de novas agendas de pesquisa (HOGAN, 2007; URICH et al., 2005; HOGAN; TOMALSQUIM, 2001), uma vez que a temática ambiental já sensibiliza boa parte das ciências sociais na região (FERREIRA, 2006a, 2006b; FERREIRA et al., 2006a, 2006b; FERREIRA et al., 2007a, 2007b; HOGAN, 2009).

Hogan (2007) ressalta que, no entanto, aparenta ser difícil mover a pesquisa sobre mudança ambiental do nível local (ou nacional) para a escala transnacional ou global, conectando-a com as discussões relativas que já se encontram consolidadas na experiência internacional, sobretudo dos países industrializados. Por outro lado, sabe-se que o financiamento de pesquisa desempenha um papel fundamental tanto na definição de agendas de investigação, como na própria organização das comunidades científicas ${ }^{9}$. A partir da análise da experiência internacional, pode-se perceber que um papel pró-ativo por parte das agências de financiamento e fomento à pesquisa é elemento determinante para impulsionar o envolvimento das ciências sociais na discussão sobre MAG (HOGAN, 2007).

Todavia, muitos países latino-americanos sofrem com a falta de recursos para investir em novas áreas de investigação e as temáticas interdisciplinares são, muitas vezes, uma das que mais sofrem com a escassez de recursos. Além disso, o financiamento para a pesquisa sobre as MAG é tradicionalmente canalizado para as ciências naturais, que têm um longo e importante acúmulo na abordagem dessas questões (URICH et al., 2005). Nesse sentido, pode-se dizer que, sem incentivos institucionais e mudanças na forma e

\footnotetext{
${ }^{8}$ A apresentação dos países segue a ordem alfabética e não corresponde à representatividade de cada país em termos de sua produção acadêmica e científica.

${ }^{9}$ Os autores agradecem aos comentários recebidos do Prof. Carlos Alfredo Joly (IB/NEPAM/UNICAMP), que chamaram a atenção para esse aspecto na fase de desenho e formulação da pesquisa.
} 
na organização do financiamento à pesquisa, os cientistas naturais só se envolverão com os cientistas sociais de forma pontual e desarticulada.

\section{Um olhar para frente: desafios para a pesquisa sobre as DHMAG}

Com o mundo cada vez mais complexo e dinâmico, o meio ambiente e os sistemas sociais estão mudando rapidamente. Água, solo e ecossistemas interagem com as MAG, configurando novos padrões e tendências de crescimento populacional, urbanização e relações com o mercado (LEACH et al., 2007). Tudo isso suscita grandes desafios em termos de pesquisa, instituições, modelos de desenvolvimento e políticas públicas na América Latina. As abordagens convencionais para essas questões na região têm ficado frequentemente restritas a fronteiras delimitadas por bases disciplinares fortes, sendo o diálogo entre as ciências naturais e sociais da região a exceção e não a regra.

Nesse sentido, a reflexão desse artigo busca contribuir com esse debate abordando alguns desafios para a pesquisa sobre as DHMAG na América Latina. Por desafios entendem-se áreas que precisam ser melhor exploradas pelos pesquisadores interessados na área de ambiente e sociedade. Para efeitos deste artigo, identificam-se quatro elementos principais ${ }^{10}$ para essa discussão que, apesar de já fazerem parte do repertório das pesquisas ambientais da região, não estão conectados à discussão sobre as MAG. São eles: ciência para sustentabilidade, escalas e abordagens multiescalares, desenvolvimento e mudanças climáticas e governança e justiça ambiental.

\section{Ciência para sustentabilidade}

A pesquisa sobre as MAG tem melhorado significativamente a compreensão da estrutura e do funcionamento da biosfera e o impacto das atividades humanas nesses processos de larga escala. A ciência da sustentabilidade é um novo campo de investigação que trabalha com as interações entre sistemas naturais e sociais, analisando como essas interações afetam o desafio da sustentabilidade de garantir as necessidades presentes e das gerações futuras, reduzindo a pobreza e conservando os sistemas de suporte à vida no planeta (CLARK, 2007). Assim, busca-se entender a condição humana e o meio ambiente a fim de atender necessidades da sociedade (KATES et al., 2001). Estes objetivos, por sua vez, exigem o diálogo entre a ciência e a esfera política.

Portanto, coloca-se como fundamental explorar novas formas de engajamento sociopolítico e entender como usar os novos conhecimentos, que emergem das novas abordagens de investigação, nos processos decisórios e na formulação de políticas públicas nos vários níveis (local, nacional, regional e global). Por exemplo, necessita-se aprofundar o entendimento sobre a variação climática e como as sociedades podem ser mais resilientes a eventos climáticos extremos (ADGER et al., 2005; LEACH et al., 2007; HOGAN, 2009). Essa ciência exige um rigor na representação do futuro, construindo cenários, encontrando novas maneiras de apresentar o futuro que levam em conta a reflexividade da sociedade a partir de atividades de pesquisas existentes (GIDDENS, 1991; BECK, 1992). A ciência para sustentabilidade demanda melhorar a compreensão dos problemas e das questões as quais a comunidade científica já está familiarizada, levando as ciências sociais a avançar rumo ao contexto da complexidade da mudança climática.

\section{Escalas e abordagens multiescalares}

A experiência acumulada mostra que a aplicação bem sucedida de abordagens multiescalares tem sido importante para melhorar a compreensão de áreas de conhecimento fundamentais para o bem-estar e a qualidade de vida da população, como saúde pública, educação, agricultura e políticas públicas ambientais (CASH et al., 2006). As escalas podem ser definidas em termos espacial, temporal, quantitativo ou mesmo representar dimensões analíticas usadas para estudar fenômenos específicos, onde os diferentes "níveis" são adotados como as unidades de análise que estão localizadas em posições diferentes de uma determinada escala (e.g. GIBSON et al., 2000).

Dessa maneira, o desafio consiste em analisar uma determinada problemática nas escalas adequadas. Muitos dos problemas ambientais globais não são apenas versões ampliadas de problemas locais. Assim, soluções dadas na

\footnotetext{
${ }^{10}$ Há muitos outros desafios que poderiam ser explorados e considerados, porém, para os propósitos desse artigo, identificamos quatro desafios a serem considerados como mais importantes para o futuro da pesquisa sobre DHMAG na América Latina.
} 
escala local não podem simplesmente serem extrapoladas para outras escalas ou níveis superiores de decisão e ação política.

Atualmente, existe uma infinidade de ferramentas metodológicas e possibilidades de abordagens multiescalares em diversos campos de conhecimento. No entanto, ainda há uma baixa compreensão dos mecanismos de interação entre as diferentes escalas, especialmente quando as análises vão além das escalas mais frequentemente estudadas (espacial e temporal, por exemplo). Pesquisadores no interior das ciências sociais com foco nos problemas ambientais devem estar conscientes das múltiplas dinâmicas multiescalares a fim de evitar uma série de obstáculos que já foram relatados pela literatura. Tais obstáculos geralmente remetem a: (1) não percepção das interações entre escalas; (2) desencontros entre escalas e níveis distintos na análise, sobretudo quando se trata de sistemas socioecológicos; e (3) diversidade de formas pelas quais diferentes atores sociais podem reconhecer e valorizar diferentes escalas em um determinado nível. Cash et al. (2006) chamaram esses desafios nas análises multiescalares de: (1) "ignorância", (2) "incompatibilidade" e (3) "pluralidade".

\section{Desenvolvimento e mudanças climáticas globais}

Na América Latina, assim como em muitos outros contextos (e.g. Ásia e África), é clara a relação entre desenvolvimento e as mudanças climáticas globais. Apesar de a região contribuir pouco em termos de emissões de GEE quando comparada aos EUA, Europa e China, por exemplo, muitos dos fatores que aparentemente agravam o problema - como aqueles decorrentes de mudanças no uso e ocupação do solo - são fortemente influenciados por atividades orientadas ao desenvolvimento econômico da região (e.g. agronegócio e grandes obras de infraestrutura).

Essas alterações acontecem em sistemas complexos e dinâmicos, que apresentam diferentes mecanismos de feedback entre os ecossistemas, a população e a economia. Para compreensão desses processos, as pesquisa devem, então, concentrar-se nos instrumentos de governança que são necessários para o encaminhamento de um determinado problema a certo nível ou escala diferente, desde que esses sejam viáveis e aceitos pela população envolvida no processo.

Dessa maneira, respostas políticas às mudanças climáticas globais na América Latina deverão estar embasadas em investigações das dimensões humanas dessas questões, considerando os diferentes atores, as múltiplas arenas de intervenção e os impactos dessas decisões não somente sobre a população, mas também sobre as localidades e os espaços geográficos. Uma perspectiva integradora das dimensões de desenvolvimento, sustentabilidade e mudança ambiental são fundamentais a compreensão dos desafios que estão colocados.

\section{Governança e justiça ambiental}

Apesar do avanço socioeconômico de alguns países na última década, a América Latina ainda é a região do mundo com a maior diferença entre ricos e pobres (UN, 2007). Soma-se a isso a necessidade de reconhecer a centralidade dos princípios de justiça ambiental para a proteção do meio ambiente e o desenvolvimento das atividades econômicas. De acordo com Acselrad et al. (2009), a crescente escassez de recursos naturais, a perda de ecossistemas importantes e as mudanças climáticas globais afetam de maneira desigual e injusta os diferentes grupos sociais, refletindo as várias formas de assimetrias sociais, políticas e econômicas nos níveis local, nacional, regional e global.

Torna-se necessário, assim, reconhecer de maneira fundamental os aspectos éticos e de justiça social como estratégia para que os impactos das MAG sejam considerados na formulação de respostas para os problemas socioambientais. Estes devem, consequentemente, estar representados nos novos arranjos institucionais que serão implementados. Nesse sentido, Biermann et al. (2009) destacam o papel das instituições e das estruturas de governança na formulação e na implementação de políticas públicas como bons exemplos de questões para investigação que poderão conduzir a melhores práticas e caminhos de desenvolvimento para a região no futuro.

\section{Conclusão}

Este artigo apresentou uma discussão sobre o estado da arte da pesquisa sobre as DHMAG na América Latina e seu desenvolvimento nas últimas duas décadas, buscando cobrir lacunas deixadas pelas poucas de tentativas de descrever e analisar essa pesquisa na região. A partir da análise de uma compilação da produção científica latino-americana organizada na forma de banco de dados, foi realizada uma 
reflexão sobre alguns dos principais desafios não só para um maior envolvimento das ciências sociais na pesquisa sobre a temática das MAG, como também para o desenvolvimento e consolidação das DHMAG na região.

Trabalhos anteriores mostraram que essa produção acadêmica e intelectual é fragmentada, sendo a área de pesquisa ainda não institucionalizada na região, embora haja um crescente número de publicações e pesquisadores dedicados aos seus temas correlatos. Tais fatos acarretam em dificuldades para a homogeneização de critérios para a análise e a avaliação dessas iniciativas (MARTINS; FERREIRA, 2009, 2010). Sugere-se que a pesquisa latino-americana sobre as DHMAG de certa forma acompanha o debate internacional, porém levando em conta especificidades regionais, como pobreza, desigualdade social, alta biodiversidade e a presença de populações tradicionais.

Nesse sentido, a dinâmica do financiamento da pesquisa parece desempenhar um papel fundamental na organização da comunidade científica e na definição de agendas de pesquisa, em particular na área de DHMAG. A experiência internacional mostra que um papel pró-ativo por parte das agências de financiamento é importante para a sensibilização das ciências sociais na pesquisa sobre dimensões humanas (HOGAN, 2007).

Como forma de contribuir com a discussão e a consolidação dessa área na região, o artigo identificou e refletiu sobre quatro desafios considerados relevantes que podem

\section{Referências}

ACSELRAD, H.; MELLO, C. C. A.; BEZERRA, G. N. O que é Justiça Ambiental? Rio de Janeiro: Garamond, 2009.

ADGER, W. N.; BROWN, K.; HULME, M. Redefining Global Environmental Change. Global Environmental Change, n. 15, p. 1-4, 2005.

ALONSO, A.; COSTA, V. Ciências Sociais e meio ambiente no Brasil: um balanço bibliográfico. BIB - Revista Brasileira de Informações Bibliográficas em Ciências Sociais, v. 53, n. 1, p. 35-78, 2002.

BECK, U. Risk Society. Towards a New Modernity. London: SAGE Publications, 1992.

The Reinvention of Politics. Rethinking Modernity in the Global Social Order. Cambridge: Polity Press, 1999. ser explorados de forma mais assertiva pela pesquisa sobre as DHMAG na América Latina: a ciência da sustentabilidade, escalas e abordagens multiescalares, desenvolvimento e mudança climática global e governança e justiça ambiental.

A partir da discussão levantada pelo artigo, algumas questões podem ser objeto de estudos e reflexões futuras, como, por exemplo, qual o impacto de redes e programas de pesquisa internacionais na adoção e na difusão de conceitos específicos no interior dessa temática? Qual é o viés disciplinar em uma área interdisciplinar como a das DHMAG? Quais as bases epistemológicas do campo das DHMAG? Essas, entre outras perguntas, podem ser objeto de trabalhos futuros, apontando caminhos importantes para investigações futuras.

\section{Agradecimentos}

Os autores agradecem os apoios recebidos da Coordenação de Aperfeiçoamento de Pessoal de Nível Superior (CAPES) e da Fundação de Amparo à Pesquisa do Estado de São Paulo (FAPESP), bem como a hospitalidade do Department of Environmental Policy Analysis (EPA), Institute for Environmental Studies (IVM), Vrije Universiteit Amsterdam, onde parte desta pesquisa foi realizada. Comentários e sugestões recebidas de um avaliador anônimo desta revista contribuíram para a melhoria do manuscrito original.

BERTERO, C. O.; CALDAS, M. P.; WOOD JR., T. Produção científica em Administração de Empresas: provocações, insinuações e contribuições para um debate local. In: ENCONTRO DAASSOCIAÇÃO NACIONAL DE PÓS-GRADUAÇÃO E PESQUISA EM ADMINISTRAÇÃO (ENANPAD), 22., 1998. Anais... Foz do Iguaçu: ANPAD, 1998.

BIERMANN, F.; BETSILL, M. M.; GUPTA, J.; KANIE, N.; LEBEL, L.; LIVERMAN, D.; SCHROEDER, H.; SIEBENHUNER, B. Earth System Governance: People, Places and the Planet. Science and Implementation Plan of the Earth System Governance Project. Amsterdam: Earth System Governance Project/IHDP, 2009. 119 p.

BRONDIZIO, E. S.; MORAN, E. F. Human dimensions of climate change: the vulnerability of small farmers in the 
Amazon. Philosophical Transactions of the Royal Society B, n. 363, p. 1803-1809, 2008.

BUTTEL, F. H. New directions in environmental sociology. Annual Review of Sociology, n. 13, p. 465-488, 1987.

Environmental and resource sociology: theoretical issues and opportunities for synthesis. Rural Sociology, v. 61, n. 1, p. 56-66, 1996.

Social Institutions and Environmental Change. In: REDCLIFT, M.; WOODGATE, G. (Orgs.). The International Handbook of Environmental Sociology. Cheltenham, UK: Edward Elgar, 1997. p. 44-54.

CASH, D. W.; ADGER, N. W.; BERKES, F.; GARDEN, P.; LEBEL, L.; OLSSON, P.; PRITCHARD, L.; YOUNG, O. Scale and cross-scale dynamics: governance and information in a multilevel world. Ecology and Society, v. 11, n. 2, p. 8, 2006.

CLARK, W. C. Sustainability Science: A room of its own. Proceedings of the National Academy of Sciences (PNAS), v. 104, n. 6, p. 1737-1738, 2007.

DRUMMOND, J. A. A primazia dos cientistas naturais na construção da agenda ambiental contemporânea. Rev. Bras. Ciênc. Soc., v. 21, n. 62, out. 2006.

DUNLAP, R.E. The Evolution of environmental sociology: a brief history and assessment of the American Experience. In: REDCLIFT, M.; WOODGATE, G. (Orgs.). The International Handbook of Environmental Sociology. Cheltenham, UK: Edward Elgar, 1997. p. 21-39.

; CATTON JR., W. Environmental Sociology. Annual Review of Sociology, v. 5, n. 25, 1979.

FERREIRA, L. C. A sociologia no horizonte do século XXI. São Paulo: Boitempo, 1997.

Ideias para uma sociologia da questão ambiental no

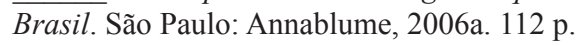

. Environmental Issues, Interdisciplinary, Social Theory and Intellectual Production in Latin America. In: ISA XVI Word Congress of Sociology. Annals... Durban: ISA, 2006b.

Teoria social, interdisciplinaridade e questão ambiental na América Latina. Campinas: Ed. UNICAMP, no prelo.

; BARBOSA, S. R. C. S.; HOEFEL, J. L. M.; FLORIANI, D.; GUIMARÃES, R.; GIESBRESCHET, M. O. A questão ambiental, interdisciplinaridade, teoria social e produção intelectual na América Latina. In: ENCONTRO DA ASSOCIAÇÃO NACIONAL DE PÓS-GRADUAÇÃO
E PESQUISA EM AMBIENTE E SOCIEDADE. BRASÍLIA: TEORIA E AMBIENTE, 3. Anais... ANPPAS, 2006a.

; GUIMARÃES, R.; FLORIANI, D.;

TAVOLARO, S. B. F. Environmental Issues, Interdisciplinary, Social Theory and Intellectual Production in Latin America. Ambiente \& Sociedade, v. 9, n. 2, p. 9-24, 2006b.

; GIESBRECHT, M. D. O.; GUIMARÃES, R.P. Resultados parciais da pesquisa "Questões Ambientais, Interdisciplinaridade, Teoria Social e Produção Intelectual na América Latina - O Banco de Dados". In: CONGRESO ASOCIACIÓN LATINOAMERICANA DE SOCIOLOGÍA. MÉXICO: TEORIA E AMBIENTE, 26. Anales... ALAS, 2007a.

; BATATA, A. G. R.; MARTINS, R. D. A. Environmental issues, interdisciplinary, social theory and intellectual production in Latin American. In: LATIN AMERICAN STUDIES ASSOCIATION LASA, 2007. INTERNATIONAL CONGRESS, 27. MONTREAL: TEORIA E AMBIENTE. Annals... LASA, 2007b.

GALLOPÍN, G. C.; FUNTOWICZ, S.; O’CONNOR, M.; RAVETZ, J. Science for the twenty-first century: from social contract to the scientific core. International Social Science Journal, n. 168, p. 219-229, 2001.

GIBSON, C.; OSTROM, E.; AHN, T.-K. The concept of scale and the human dimensions of global change: a survey. Ecological Economics, n. 32, p. 217-239, 2000.

GIDDENS, A. As consequências da modernidade. São Paulo: Ed. UNICAMP, 1991.

Press, 2009.

The Politics of Climate Change. Cambridge: Polity

GRANT, W. E.; PETERSON, T. R.; PETERSON, M. J. Quantitative modelling of coupled natural/human systems: simulation of societal constraints on environmental action drawing on Luhmann's social theory. Ecological Modelling, n. 158, p. 143-165, 2002.

HANNIGAN, J. A. Environmental Sociology. A social constructionist perspective. London/NY: Routledge, 1995.

HOGAN, D. J. Human Dimensions of Global Environmental Change. Ambiente \& Sociedade, v. 10, n. 2, p. 161-166, 2007.

População e mudanças ambientais globais. In: HOGAN, D. J.; MARANDOLA JR., E. (Orgs.). População e mudança climática. Dimensões humanas das mudanças ambientais globais. Campinas: Núcleo de Estudos de População (NEPO/UNICAMP), 2009. 292 p. 
; MARANDOLA JR., E. (Orgs.). População e mudança climática. Dimensões humanas das mudanças ambientais globais. Campinas: Núcleo de Estudos de População (NEPO/ UNICAMP), 2009. 292 p.

; TOLMASQUIM, M. T. Human Dimensions of Global Environmental Change: Brazilian Perspectives. Rio de Janeiro: Academia Brasileira de Ciências (ABC), 2001.

INTERNATIONAL GEOSPHERE-BIOSPHERE PROGRAMME (IGBP). Global Change and the Earth System: a Planet under Pressure. IGBP Science 4. Stockholm: IGBP, 2001.

INTERNATIONAL HUMAN DIMENSIONS PROGRAMME (IHDP). Strategic Plan 2007-2015: Framing Worldwide Research on the Human Dimensions of Global Environmental Change. Bonn: IHDP, 2007.

INTERNATIONAL PANEL ON CLIMATE CHANGE (IPCC). Summary for policymakers. Genebra: IPCC, 2007.

JANSSEN, M. A.; SCHOON, M. L.; KE, W.; BORNER, K. Scholarly networks on resilience, vulnerability and adaptation within the human dimensions of global environmental change. Global Environmental Change, v. 16, p. 240-252, 2006.

KATES, R. W.; CLARK, W. C.; CORELL, R.; HALL, J. M.; JAEGER, C. C.; LOWE, I.; MCCARTHY, J. J.; SCHELLENHUBER, H. J.; BOLIN, B.; DICKSON, N. M.; FAUCHEUX, S.; GALLOPIN, G. C.; GRÜBLER, A.; HUNTLEY, B.; JÄGER, J.; JODHA, N. S.; KASPERSON, R. E.; MABOGUNJE, A.; MATSON, P.; MOONEY, H.; MOORE III, B.; O'RIORDAN, T.; SVEDIN, U. ENVIRONMENT AND DEVELOPMENT: Sustainability. Science, v. 292, n. 5517, p. 641-642, 2001.

LAHSEN, M.; NOBRE, C. Challenges of connecting international science and local level sustainability efforts: the case of the Large-Scale Biosphere-Atmosphere Experiment in Amazonia. Environmental Science and Policy, n. 10, p. 62-74, 2007.

LEACH, M.; SCOONES, I. STIRLING, A. Pathways to Sustainability: an overview of the STEPS Centre approach. STEPS Approach Paper. Brighton: STEPS Centre, 2007.

LIMA, G. F. C.; PORTILHO, M. F. A sociologia ambiental no contexto acadêmico norte-americano: formação, dilemas e perspectivas. Teoria \& Sociedade, n. 7, p. 241-276, 2001.

LOWE, P.; RUDIG, W. Political Ecology and the Social Sciences - The State of the Art. British Journal of Political Science, n. 16, p. 513-550, 1986.
MARTINS, R. D. A; FERREIRA, L. C. Assessing the Research on Human Dimensions of Global Environmental Change in Latin America. Teoria \& Pesquisa, v. 18, n. 2, p. 31-52, 2009.

.The research on human dimensions of global environmental change in Latin America: Looking back, moving forward. International Journal of Climate Change Strategies and Management, v. 2, p. 264-280, 2010.

MILLENNIUM ECOSYSTEM ASSESSMENT (MEA). Ecosystems and Human Well-being: Current State and Trends, Volume 1. Washington: World Resources Institute, 2005.

MOL, A. P. J.; SPAARGAREN, G. Ecological modernisation theory in debate: a review. Environmental Politics, n. 9, p. 17-50, 2000.

NATIONAL RESEARCH COUNCIL (NRC). Global environmental change: research pathways for the next decade. Washington D.C.: National Academy Press, 1999a.

. Human Dimensions of Global Environmental Change: Research Pathways for the Next Decade. Washington D.C.: National Academy Press, 1999b.

PULIDO, L. A Critical Review of the Methodology of Environmental Racism Research. Antipode, v. 28, n. 2, p. 142-159, 1996.

REDCLIFT, M.; WOODGATE, G. (Orgs.). The International Handbook of Environmental Sociology. Cheltenham, UK: Edward Elgar, 1997.

ROCKSTRÖM, J.; STEFFEN, W.; NOONE, K.; PERSSON, A.; CHAPIN III, F. S.; LAMBIN, E. F.; LENTON, T. M.; SCHEFFER, M.; FOLKE, C.; SCHELLNHUBER, H. J.; NYKVIST, B.; de WIT, C. A.; HUGHES, T.; van der LEEUW, S.; RODHE, H.; SÖRLIN, S.; SNYDER, P. K.; COSTANZA, R.; SVEDIN, U.; FALKENMARK, M.; KARLBERG, L.; CORELL, R. W.; FABRY, V. J.; HANSEN, J.; WALKER, B.; LIVERMAN, D.; RICHARDSON, K.; CRUTZEN, P.; FOLEY, J. A. A safe operating space for huminaty. Nature, v. 461, p. 472-475, 2009.

STEFFEN, W.; SANDERSON, A.; TYSON, P.; JÄGER, J.; MATSON, P.; MOORE III, B.; OLDFIELD, F.; RICHARDSON, K.; SCHELLNHUBER, J.; TURNER II, B. L.; WASSON, B. Global Change and the Earth System: A Planet Under Pressure. Berlin: Springer-Verlag, 2004.

UNITED NATIONS (UN). Local Governments'International Mid Term Evaluation Conference on the Millennium Development Goals (MDGs). Rome: June, 2007. 
MARTINS, R. A.; FERREIRA, L. C. Desafios para a pesquisa sobre as dimensões humanas das mudanças...

URICH, P.; CAMPBELL, J.; GILLESPIE, A.; SCRIMGEOUR, F. Human Dimensions of Global Environmental Change: A Review of Frameworks and Research Gaps. The CLIMPACTS Programme. IGCI Report for FRST, 2005.
WILBANKS, T. J.; KATES, R. W. Global Change in Local Places: How Scales Matters. Climatic Change, n. 43, p. 601$628,1999$.

Recebido em setembro de 2010. Aceito em dezembro de 2010. Publicado em junho de 2011. 